\title{
Interculturalidad: miradas múltiples. Desafío Creativo Cooperativo
}

\section{Doria, Patricia M. [ver currículum del autor, docente de la Facultad de Diseño y Comunicación]}

\section{Resumen:}

En el área de Diseño, los proyectos son individuales, creativos, y personales generando mucho énfasis en la enseñanza, el aprendizaje y la corrección grupal por parte del profesor, que debe respetar el estilo personal de cada alumno. El sentido es fomentar las miradas múltiples y diferentes, aceptar las diversidades e influencias interculturales, y de este modo citar en las producciones las improntas culturales para nutrir y, generar diseños de mayor originalidad para acceder a una mayor competitividad. A partir de estas ideas se generó una técnica específica para áreas del Diseño donde los proyectos son el eje central y directriz de la formación de los alumnos; esta técnica es denominada Desafío Creativo Cooperativo (DCC).

Palabras clave:

Proyecto - aprendizaje - cooperativo - creatividad - interculturalidad - diseño - desafío.

Introducción La necesidad de formación en técnicas de aprendizaje cooperativo ha sido y es, un hecho relevante en la formación de alumnos universitarios porque supone un desplazamiento de una educación centrada en la enseñanza hacia una educación centrada en el aprendizaje, a la vez que se exige a los alumnos nuevas competencias interpersonales que facilitan la interacción social y la cooperación. Esta nueva interacción social educativa surge en la Argentina a partir del año 2001, donde se observa el ingreso de muchos estudiantes de países Latinoamericanos, lo que genera replantear el sentido del término interculturalidad que es inseparable de la cuestión de la identidad. El hecho de relacionarse de manera simétrica con personas, saberes, sentidos y prácticas culturales distintas, requiere un autoconocimiento de quién es uno, de las identidades propias que se 
forman y destacan tanto lo propio como las diferencias. La identidad propia no es algo que podemos elegir, sino algo que se tiene que negociar socialmente con todos los otros significados e imágenes construidos como conocimientos que nuestro propio uso de la identidad activan (Hall, 1997). Sin embargo, y a pesar del contacto y relación cultural y su naturaleza cambiante, los grupos culturales siguen construyendo, reinventando y manteniendo algo propio que los distinguen de otros grupos y desafían nociones de una mezcla generalizada y homogenizante. Esto se evidencia con claridad en la Universidad y, específicamente, en las carreras de Diseño donde estas culturas diferentes se ponen en evidencia a través de la conceptualización propia de las formas, texturas y colores. Estos conceptos nos hablan de historia, idiosincrasia, tradición, mitos y leyendas propias de cada pueblo, ciudad o país. Por este motivo, es muy importante el trabajo de enseñanza cooperativa dentro del aulataller, para que los alumnos de diversas extracciones no se sientan excluidos -sino todo lo contrario- de los proyectos de diseño donde se trabaje la totalidad y la diversidad. No existe una receta que permita implementar un proyecto de gestión del conocimiento en las diferentes instituciones educativas. Cada espacio académico es particular y requiere un abordaje específico. Con este trabajo, pretendo determinar algunas consideraciones acerca de las interrelaciones entre el diseño, su enseñanza y aprendizaje, con un análisis de las etapas por las cuales transitan los alumnos de diseño, y la aplicación de la técnica propuesta, como simulacros de experimentación, con comitentes reales -que son sus propios compañeros de aula que se convierten en colegaalumno- otorgando diferentes niveles en el aprendizaje del diseño a través de la implementación de la técnica de aprendizaje denominada "Desafío Creativo Cooperativo" (DCC). La enseñanza en una clase de diseño se desarrolla a partir del producto completo denominado el "proyecto", en el cual se vincula el material y los límites o contornos formales de los diseños que va a contar con diferentes etapas de aprendizaje. Es decir, la necesidad de la concreción de este producto-proyecto es una condición ineludible para la comprensión del proceso proyectual. Shön (1992) indica:

En su sentido más amplio, el diseño implica complejidad y síntesis... A través del estudio de estos temas en el contexto de los talleres de diseño arquitectónico, esquematizaré los rasgos principales de un practicum reflexivo aplicable a la preparación para el arte en otras profesiones... Los estudiantes aprenden haciendo y sus instructores funcionan más como tutores que como profesores... (Shön, 1992)

El alumno en una primera etapa trata de controlar las variables propias del proyecto, a medida que avanza en el desarrollo se va adentrando más y más en la problemática a resolver, ya que el conocimiento-aprendizaje es netamente personal y su validez se acentúa a través del compromiso, los valores y sensaciones particulares. La forma comienza a delinearse y a constituirse en una entidad significativa, ideada como un producto cultural, histórico, estético e inserto en un ámbito que posibilita su existencia. Es decir, esta construcción pasa a ser un signo, con una carga de significado, y a partir de allí pasa a transformarse en un vehículo para la constitución de la totalidad de un sistema de aprendizaje; y en este punto ingresa la mirada valorativa de sus colegas-alumnos. Ninguna acción en diseño es irreversible, el alumno en el proceso puede probar, observar y probar de nuevo. Los proyectos no son simples dibujos, son la manifestación poética y constructiva de su creatividad, la que debe ser estimulada a través de diferentes ejercicios con consignas claras para el desarrollo posterior del mismo. Se trata de una parte del proceso real de producción de un diseño; donde el estudiante puede detener el proceso de diseño, pensar sobre lo que está haciendo y volver a realimentar el trabajo con una nueva mirada. En el transcurso del proyecto, se van desarrollando secuencias de aprendizaje donde se comienza a aplicar esta técnica de instrucción denomina de "Destreza Creativa Cooperativa", en la cual se pueden corregir los errores y contemplar las opiniones de los colegasalumnos, en el grupo. El atractivo de realizar con los alumnos proyectos 
de simulación de la realidad, se relaciona con la posible utilización de un conocimiento compartido con el grupo; al crear un contexto real, permite compartir experiencias entre los distintos proyectos propuestos. Al otorgarle un carácter activo y participativo a la experiencia se facilita la interacción y la elaboración colectiva del conocimiento, permitiendo que el mismo sea construido e integrable al ya existente. Estos espacios de simulacro son espacios de elaboración compartida del conocimiento, permitiendo generar modelos de estas prácticas casi reales, con diálogos abiertos entre colegas-alumnos, donde se debate la propuesta. Todos opinan y el profesor logra un clima de trabajo e interacción similar a lo que ocurre en un estudio de diseño, colaborando de este modo a una práctica casi real para que luego fuera de la Universidad, se inserten directamente en ellas. Este vínculo interactivo entre los miembros del grupo permite una colaboración constructiva; en estas evaluaciones y discusiones grupales van aprendiendo a formularse preguntas y a buscar sus respuestas en forma sistemática, estableciendo un pensamiento crítico sobre el desarrollo de su propio trabajo y del resto de sus compañeros; pero dichas críticas serán realizadas en forma constructiva, es decir, siguiendo los mismos principios que él o ella esperan que sean usados en su propia evaluación. Este programa en su construcción genera una lógica de interconexión entre formaciones proyectuales, y conocimientos diversos, sobre temas a fin al Diseño de Indumentaria. A través de la articulación del programa, logramos darle un orden, lógica y estructura al pensamiento creativo. La realidad de cada institución define la dinámica y el camino a seguir, y esto nos otorga un contexto regulado con una estructura, que no deja de lado la originalidad en la innovación propuesta, en la circulación del conocimiento y en las demandas disciplinares. Según Pérez Lindo en su escrito ¿Para qué educamos hoy? indica:

Lo importante no son las etiquetas sino el reconocimiento de que los fenómenos educativos son multidimensionales, que dependen de actores, ideas, procesos, y estructuras. Como cualquier fenómeno social. Pero acontece con la educación que al mismo tiempo que reproduce las condiciones de una sociedad tiene la capacidad para transformarla. El solo hecho de adquirir una competencia lingüística modifica nuestra subjetividad. El solo hecho de aprender a pensar modifica nuestra estructura mental. (Pérez Lindo, 2009, p. 4)

Los docentes en general, en sus prácticas cotidianas comienzan a estar cómodos y acostumbrados con sus saberes ya adquiridos, probados y aplicados en el aula. Los sistemas educativos modernos no han ayudado en este sentido porque han acentuado las compartimentaciones disciplinarias y la fragmentación de las visiones del mundo. Siguiendo a Pérez Lindo, él anticipa que para construir una nueva visión compleja de los procesos educativos debemos empezar por reconocer que han entrado en crisis la idea de la realidad, la idea de la verdad, los paradigmas sobre la subjetividad, la visión de los entornos naturales y culturales, es decir los principios que guiaban los métodos de enseñanza. Por estos motivos, es bueno que el conocimiento se convierta en el pilar fundamental para solucionar las dificultades que se presenten, fortalecer lo acertado y mediar nuevos procesos para realizar las mejoras pertinentes. Para nuestros fines del conocimiento en este proyecto podemos tener en cuenta a los autores, Rodríguez, La Matta, y Gutiérrez (2001, p. 17), que según ellos "la gestión del conocimiento es una combinación organizada y estructurada de ideas e información”. La Universidad parece estar perdiendo posiciones como centro de generación de conocimiento; pero por otro lado, se puede visualizar que la solución a este problema pasa porque la Universidad realice mayores esfuerzos por orientar su investigación a la aplicación del conocimiento científico-técnico en colaboración con las instituciones educativas y las administraciones públicas, como indica el modelo de triple hélice planteados por Etzkowitz y Leydesdorff (1995). Por otro lado, Nonaka \& Takeuchi (1995), definen también a la gestión del conocimiento como la transformación de conocimiento explícito en conocimiento tácito y viceversa, a partir de prácticas de 
combinación (de explícito a explícito), interiorización (de explícito a tácito), socialización (tácito para tácito) y exteriorización (tácito a explícito). La asignatura Diseño es la troncal de las carreras de Indumentaria, pero esta asignatura no contiene una doctrina latente, sino que es un aprender haciendo, en un doble sentido: primero se aprende a diseñar objetos; segundo se aprende sobre "algo" en el ejercicio mismo de ese algo. No es un conocimiento discursivo, sino uno que se valida en la práctica. En general, en la práctica los proyectos son individuales, creativos, y personales, donde el profesor hace mucho énfasis en el estilo personal de cada alumno. El sentido es fomentar las miradas múltiples y diferentes, incluir las diversidades en las influencias, citando las improntas culturales y de este modo marcar la diferencia, generar productos originales y ganar en competitividad. La idea central es generar una técnica específica para áreas donde los proyectos son el eje central y directriz de la formación de los alumnos, esta técnica es denominada Desafío Creativo Cooperativo (DCC). Las etapas directrices de esta técnica se centra en el proceso de aprendizaje proyectual y se estructura a través de:

- Destreza: La destreza hace referencia a todos los aspectos de habilidad manual con diferentes materiales, a la utilización competente de determinados medios tecnológicos, a la facilidad para utilizar y transformar materiales, a la comprensión y aplicación de nuevos conceptos y aplicaciones. - Metodología: La metodología incluye la evolución gradual del estilo individual de trabajo, la habilidad para planificar, estructurar y evaluar el propio proceso de trabajo, la capacidad de combinar la experimentación sistemática con el pensamiento intuitivo, y la habilidad para organizarse. - Conocimiento: El conocimiento, incluye historia, arte, cultura, estética, ética, comunicación. Además, para el área del diseño los futuros profesionales deberán estar capacitados con conocimientos y una comprensión profunda de la interdependencia entre sociedad, política, economía y ecología.

Estas definiciones ayudan a entender que tanto a nivel individual como a nivel grupal las organizaciones/instituciones deben realizar una serie de procesos como la creación, adquisición, identificación y transferencia del conocimiento, lo que permite llegar a la consecución de un objetivo específico. De esta forma, se fortalecen los espacios dinámicos de acción en el aula, en donde se da la mayor innovación y por lo tanto, es previsible un mayor grado de anarquía, diríamos que es una anarquía controlada. El conocimiento, se define como el proceso constante para identificar, encontrar, clasificar, proyectar, presentar de un modo más eficiente el conocimiento y la experiencia, de forma que mejore el alcance del empleado para conseguir ventajas competitivas. (Lahaba y Santos, 2001, p. 123). Son conocidos los 5 modelos de técnicas de enseñanzaaprendizaje: Coop Coop, Cooperación Guiada, Grupos de Investigación, Concurso y Puzzle. Todos estos ejemplos de técnicas son de fácil aplicación en áreas teóricas, pero a partir del nuevo abanico de carreras netamente proyectuales que han surgido en la Argentina y son tan convocantes para estudiantes de Latinoamérica, como son los diseños, estas técnicas deben renovarse para su aplicación en áreas donde el pensamiento sistémico-proyectual, e individual pasa a ser su pilar fundamental. Por esta razón este nuevo enfoque sobre el conocimiento, puede ser tomado como la clave fundamental para un futuro exitoso, es un movimiento que nace en el interior del claustro hacia los mismos docentes. El compartir el conocimiento: otorga como objetivo la transferencia del mismo al lugar donde sea necesario, en el momento adecuado y con la calidad requerida. Por lo tanto, el conocimiento se debe distribuir para que otras personas puedan encontrarlo y lo utilicen en procesos que añadan valor. La mayoría del conocimiento se transmite de mejor forma de persona a persona, mediante la colaboración de talleres, aprendizaje en el trabajo diario, etc. El poder utilizar el conocimiento: añade valor solamente cuando se utiliza en la empresa o institución; determina las necesidades 
de la institución a este respecto, y debe servir como referencia para la creación, almacenamiento y las formas de compartir conocimiento. La gestión del conocimiento en una institución ayuda a identificar, adquirir, desarrollar, compartir, utilizar, la información, las opiniones, experiencias y puntos de vista que aportan todos los integrantes del programa.

Cómo se aplica la gestión del conocimiento en el aula-taller. Para aplicar la gestión del conocimiento, se tiene que crear y fomentar el espíritu de equipo entre alumnos-maestros de procedencia diversa y formaciones también diversas. Pero al mismo tiempo, es imprescindible crear un sistema de externalización e intercambio de experiencias. La herramienta perfecta son las clases prácticas, a las que se traslada el principio básico de la Gestión del Conocimiento: "lo que una organización y sus empleados conocen es la base esencial del funcionamiento de la organización" (Davenport y Prusak, 2001 p. 9). Esta gestión del conocimiento, busca los siguientes beneficios específicos:

- Transferir de la forma más rápida y efectiva el conocimiento adquirido en el aula, para que la información no pierda su valor. - Utilizar al docente como motor de la innovación; que es la fuente principal interna. - Identificar nuevas estrategias de experimentación y solución a los problemas. - Transformar el conocimiento de los docentes en competencias corporativas y ventajas competitivas. - Medir y compartir los efectos de la transferencia de conocimiento entre docentes. - Aprender con mayor apertura y darle mejor provecho a la experiencia entre colegas. - Capitalizar el conocimiento creado, y lograr un retorno en la inversión en capacitación del personal y actualización en los syllabus de las asignaturas proyectuales. - Crear en la organización una cultura de mejora y aprendizaje continuo

Para crear espíritu de equipo, se estableció que el objetivo de los trabajos de diferentes docentes debían ser comunes: entre todos se iba a elaborar un manual de diagnóstico organizativo (un depósito de conocimiento), compartiendo la responsabilidad de la calidad del resultado final. Cada equipo docente/alumno debe trabajar un problema relacionado con la experiencia de la asignatura en la que son considerados expertos. En palabras de Kark Weick (1995. pp. 60-61) citado en Davenport y Prusak la respuesta es:

(...) algo que preserve lo plausible y coherente; algo que sea razonable y fácil de memorizar; algo que abarque la experiencia previa y las expectativas; algo que resuene en otras personas; algo que pueda ser interpretado en retrospectiva, pero que también se pueda usar en perspectiva; algo que capture tanto sentimientos como pensamientos; algo que permita que las mejoras encajen en las imperfecciones actuales; algo que sea interesante proyectar. En resumen, lo que es necesario para que algo tenga sentido. (Davenport y Prusak, 2001, p. 95)

Esta capacitación genera el ámbito propicio para dichas competencias curriculares. El conocimiento es inseparable de las prácticas, es imposible conocer sin hacer. La potencialidad de este aprendizaje está ligada a la potencialidad de cada uno de los saberes puestos en escena por los participantes al programa. Este programa de capacitación otorgará la competencia de aprender y generar conocimientos, y será la médula troncal; porque los cambios pueden ser interpretados como la medida de transformación y actualización sobre los valores, creencias y postulados compartidos, por los docentes participantes. Drucker (2004) manifiesta que para transformar datos en información hacen falta conocimientos, estos conocimientos se generan a través de una sinergia cognitiva entre los participantes. Estas capacitaciones implican un cambio en el sistema de actualización, planificación, información cruzada y evaluación, obligando a explorar nuevas estrategias de 
aprendizaje modificando y actualizando los sistemas de formación docente. Este conocimiento adquirido se manifiesta a través de las denominadas reglas empíricas, que conforman guías flexibles para la acción que se desarrolla en este Programa a través de distintos medios: por el método de ensayo y error, por la experiencia y la observación, de forma heurística. En cualquiera de estas etapas del Programa damos cuenta del estado del proceso que vivencia el alumno-maestro, en el aprendizaje en el aula taller; en un espacio donde se aprenden técnicas a partir de un marco teórico, fluido entrelazado con la creación y el aprendizaje, en el cual se mezclan experiencias, sensaciones, emociones y pensamientos, a los efectos de una mayor comprensión del acto creativo, donde las morfologías se ven afectadas por el lenguaje del diseño. En la universidad estas prácticas fundamentan estas nuevas acciones en pro de un nuevo conocimiento, que otorga pertenencia académica y de inter-cátedras capitalizando los saberes propuestos en las planificaciones académicas. Esto lo podemos definir y articular a través de dos flujos de conocimiento: por un lado el flujo de exploración, que es el conocimiento desde el individuo hacia el grupo y hacia la organización y representa los flujos que permiten el desarrollo y la asimilación de nuevos conocimientos entre cátedras, modificando las creencias y comportamientos adquiridos en el pasado. Están por tanto orientados a la renovación, la creación, la variación y el cambio. Y por el otro, el de exploración, que es el conocimiento del pasado desde la organización al grupo y al docente-maestro. Son los flujos que se difunden, combinan y utilizan aquellos conocimientos que ya forman parte de las estructuras de conocimiento a través de los diferentes niveles de la organización del programa.

\section{Esquema de implementación}

Profesor:

1. Explica las consignas del ejercicio de Diseño, motivación intrínseca. 2. Un alumno expone su trabajo. Guía de consignas precisas. 3. Debate, discusión de los estudiantes en la clase, guiados por el profesor. 4. El grupo se acerca en semi - círculo al alumno que presenta el proyecto. 5. El docente conduce la corrección/aprendizaje, elige a 2 o 3 alumnos colegas. 6. El docente comienza a preguntar a los alumnos - colegas sobre el trabajo del alumno que expone delante del grupo realizando la presentación. 7. Los alumnos guías comienzan a explicar el trabajo como si fuera propio, generación de diálogo con fundamentaciones teóricas válidas. 8. Entre ambos alumnos colega y expositor: logran una síntesis. 9. Se evalúa al alumno que presenta y a los que ocupan los lugares de alumnos colegas defensa del proyecto. 10.Se anima a los alumnos colegas a descubrir y expresar sus intereses sobre la propuesta de los proyectos presentados.

Este ida y vuelta entre colegas-alumnos ayuda al alumno (expositor) a reconocer los límites que tienen los medios visuales, siendo utilizados como experimentación para concederle al futuro diseñador la posibilidad de eliminar aquellos elementos del mundo real que pudieran entorpecer su desarrollo experimental y en el proceso legitimar las ideas de diseño. En este recorrido se pueden apreciar múltiples beneficios que aporta la enseñanza los cuales logran adquirir actualidad, vigencia en referencia a los aportes estéticos-formales, y al escuchar al grupo como comitentes reales, pueden aplicar nuevas experimentaciones, tecnologías, manufacturas y producción para transmitir un significado, una poética propia e individual. La enseñanza del diseño se basa en la relación docente-alumno-producto; así comienza el conocimiento a tomar cuerpo real y concreto, pero en esta tríada se incorpora una nueva mirada.

Competencias a desarrollar en los estudiantes. Resultados esperados 
El profesor debería poder:

- Verificar si el alumno ha logrado apropiarse positivamente de los saberes propuestos. - Evaluar si la devolución por parte del profesor a cargo del grupo y de sus pares (oficiando de comitentes reales), brinda elementos de mejora al producto realizado por el alumno expositor. - Motivar la discusión a través de los objetivos propuestos. - Brindar elementos para que el alumno sepa si su aprendizaje ha sido significativo o no, a través de la mirada creativa de otro alumno participante del grupo y puede proseguir en el crecimiento del proyecto - Evaluar si se registra un intercambio intercultural entre alumnos de diferentes países. - Reconocer las diferentes realidades culturales y apreciaciones estéticas diversas, desarrollando la capacidad de integración. - Verificar las visiones críticas como contribución al desarrollo creativo del proyecto de los alumnos. - Desarrollar la capacidad de integración, de conocimiento y aceptación propia y del entorno. - Desarrollar las competencias y habilidades necesarias para que el planteamiento del ejercicio de diseño resulte bien enfocado y dirigido - Fomentar la personalidad relevante, distintiva, con una visión crítica y de respeto ante sus proyectos y sobre las devoluciones realizadas de sus colegas-alumnos.

De tal manera, estas competencias interculturales darán lugar a la disposición y ejercicio, por parte del alumno, con una serie de conocimientos, habilidades y actitudes que le permitan el desarrollo de su tarea o función, sea la que fuere su cualificación de origen, le permitirán relacionarse y superar los posibles retos y dificultades que puedan surgir debido a las interacciones culturales. Según Santos Rego:

Hablar de educación intercultural es hablar de educación inclusiva y esto implica defender radicalmente la idea de la inclusión de todos y de una convivencia pacífica y en libertad, lógicamente no exenta de conflictos y vicisitudes en una escuela plural, compleja y en permanente cambio social y cultural. (Según Santos Rego, 2013, p. 124)

Esta nueva Técnica DCC consiste en trabajar juntos para alcanzar objetivos comunes. En este modelo de situación cooperativa, los alumnos procuran obtener resultados que sean beneficiosos para ellos mismos y puedan ser decodificados para todos los demás alumnos integrantes en el aula. A través de la información disponible se realizará la implementación, con los docentes participantes de las áreas troncales afines, y con distintas gradientes de complejidad curricular, para que la capacitación se nutra de diferentes conocimientos; el espacio físico donde se desarrollará la capacitación es conveniente que sea en el aula-taller donde se realizan las prácticas con los estudiantes y se vivencian las asignaturas proyectuales. La gestión del conocimiento no sólo se logra con el respeto a los procesos de gestión general, sino a partir de los logros de los colectivos y los equipos, que aportan el conocimiento específico y la inteligencia. Se optimizará el conocimiento y se compartirá el estilo y la cultura que cada uno posee a partir de las variables que se analicen en la ejercitación áulica. El factor confianza es preponderante para el buen desarrollo de este proyecto, estos encuentros cara a cara y con diferentes roles nos lleva a el punto más importante que es escuchar el conocimiento del otro y ponerlo en práctica en un proyecto, la escucha y el dejarse influir por lo que nuestros colegas manifiestan. Los conocimientos aprendidos en estas prácticas tienen varios niveles (nivel de comprensión, nivel de aplicación, y nivel sistémico). Estas variaciones generan una malla curricular para cada uno de los conocimientos

\section{Obstáculos}


A partir de este Proyecto, nos encontramos entonces ante el incipiente desarrollo y crecimiento de una nueva área de capacitación que adicional a su particularidad de permeación permite un amplio abanico de posibilidades temáticas en otras áreas del conocimiento, y presenta una clara necesidad en la consolidación de procesos de capacitación alrededor de nuevas e innovadoras prácticas de formación docente. Entre los obstáculos posibles en la implementación, detectamos que existe una inercia a los aprendizajes ya aprendidos y que se tiende a la repetición casi inconsciente de los saberes. También es claro que en general los docentes luego de varios años en su tarea comienzan a tender a una carencia de permeabilidad para reconocer y tomar en cuenta otros puntos de vista; y en algunos casos, oponen resistencia a compartir sus ideas y experiencias con pares; cuando en realidad el conocimiento se enriquece y ensancha al ser compartido. Esta capacitación pretende crear un conocimiento compartido que es muy difícil en ocasiones de implementar y se presenta como complejo en la adopción de ideas que surgen del núcleo interno académico.

\section{Resultados esperados}

En este nuevo modelo de programa, en esta nueva gestión del conocimiento se presentan dos procesos fundamentales: uno es la creación de un conocimiento construido entre pares y compartido por los mismos participantes y el otro, la transmisión al aula de este nuevo conocimiento. La pretensión de este nuevo Programa es que sea tenido en cuenta como una estrategia en la búsqueda de nuevos conocimientos de prueba aprendizaje / prueba, error. Y que su aplicación optimice el aprendizaje a través de nuevas estructuras construidas a medida a partir de formaciones académicas similares, pero con culturas, vivencias y estilos diferentes. Esto llevará a realizar una revisión del conocimiento de todo el grupo docente participante e involucrado en el proyecto. Puntos a tener en cuenta como resultados esperados luego de la capacitación:

- Se pone en valor el conocimiento explícito. - Se valora el conocimiento de la experiencia. - Se valoran los conocimientos colectivos. - Se enfatiza "la escucha". - Se aplica en el aula y el estudiante percibe un consenso creativo sobre los diferentes saberes impartidos. - Se realiza una auto-evaluación permanente del Programa de Capacitación para analizar sus beneficios para los estudiantes en el aula. - Se enfatiza en un enfoque de miradas múltiples tomando distancia del "pensamiento único", tendiendo a fortalecer la disciplina, flexibilizando los planes de estudios y superando la visión mono-disciplinaria de las cátedras.

\section{Conclusiones}

El aprendizaje cooperativo marca el empleo didáctico de grupos reducidos en los que los alumnos trabajan juntos para maximizar su propio aprendizaje y el de los demás. Pero desde el lugar del debate y la corrección a través de miradas múltiples. En este caso se da un aprendizaje creativo y cooperativo y esto le permite al docente alcanzar varias metas importantes al mismo tiempo. En primer lugar, lo ayuda a elevar el rendimiento de todos sus alumnos, incluidos tanto los especialmente dotados como los que tienen dificultades para aprender. En segundo lugar, lo ayuda a establecer relaciones positivas entre los alumnos, sentando así las bases de una comunidad e aprendizaje en la que se valore la diversidad. En tercer lugar, les proporciona a los alumnos las experiencias que necesitan para lograr un saludable desarrollo social, psicológico y cognitivo. En cuarto lugar, les brinda a los alumnos una capacidad de escucha, y de unión de criterios de corrección. Parte del atractivo de realizar con los alumnos proyectos de simulación de la realidad, se relaciona con la posible utilización de un conocimiento compartido; al crear un contexto real, permite compartir experiencias entre los distintos proyectos propuestos; al darle un carácter activo y participativo a la experiencia, facilita la interacción y la elaboración 
colectiva del conocimiento, permitiendo que el mismo se construido e integrable al ya existente. Estos espacios de simulacro con la mirada de colegas que son pares, se transforman en espacios de elaboración compartida del conocimiento, y proponen desafíos de la creatividad, permitiendo generar modelos de estas prácticas, logrando que se inserten directamente en ellas. Esta interacción entre los miembros del grupo permite una colaboración constructiva. Todos en el aula opinan sobre el desarrollo del proyecto de todos. En estas evaluaciones grupales van aprendiendo a formularse preguntas y a buscar sus respuestas en discusiones grupales en forma sistemática, estableciendo un pensamiento crítico sobre el desarrollo de su propio trabajo y del resto de sus compañeros; pero dichas críticas serán realizadas en forma constructiva, es decir, siguiendo los mismos principios que él o ella esperan que sean usados en su propia evaluación. En estas relaciones interculturales es necesario conocer el impacto de la cultura y las relaciones interculturales en dichos comportamientos profesionales.

\section{Bibliografía}

Arceo, F., Rojas, G. y González, E. (2002). Estrategias docentes para un aprendizaje significativo: una interpretación constructivista. McGraw-Hill. Cardozo A. (s.f) Hacia la Internacionalización de la Educación superior. Recuperado el: 07 de junio de 2015. Disponible en: www.mineducacion.gov.co/1621/articles230245_archivo_pdf_articulo_internacionalizacion.pdf. Chiavenato, I. (2004). Comportamiento organizacional. International Thomson. Dagnino, R. y Thomas, H. (1999). La política científica y tecnológica en América Latina; 1971. En REDES, Vol. 6 No 13. Buenos Aires. Davenport, T. H., y Prusak, L. (2001). Conocimiento en acción como las organizaciones manejan lo que saben. Prentice Hall. Drucker, P. (2004). La disciplina de la innovación. Harvard Business Review América Latina. Duderstadt, J. y Orzanco, M. (2010). Una universidad para el siglo XXI. Cátedra UNESCOUNU" Historia y Futuro de la universidad. Buenos Aires: Universidad de Palermo. Etzkowitz, H. y Leydesdorff, L. (1995). The triple helix of university industry government relations: a laboratory for knowledge based economic development. EASST Re-view 14, No 1, p.p. 11-19. Gacel - Ávila, J. (2003). La internacionalización de la educación superior: paradigma para la ciudadanía global. Guadalajara: Universidad de Guadalajara. Harvard Business Review. (2003). Gestión del Conocimiento. España: Deusto.

Hall, S. (1997). Representation: Cultural representations and signifying practices. Sage. Johnson, D., Johnson, R., y Holubec, E. (1999). El aprendizaje cooperativo en el aula. Barcelona: Paidós. Jalón, M. (2003). Educación intercultural y aprendizaje cooperativo. Ediciones Pirámide. Kenneth G. (1992). El yo saturado. Dilemas de identidad en el mundo contemporáneo. Barcelona: Paidós. Khun, M. y Mcportland, T. (1954). And empirical investigation of self-ttitudes. American Sociological Review, 19, pp. 58-76. Lahaba, N., y Santos, M. (2001). La gestión del conocimiento: una nueva perspectiva en la gerencia de las organizaciones. Acimed, 9 (2), pp. 121126. Lahaba, N. y Santos, M. (2001). La influencia de los paradigmas mentales en la gerencia moderna. Un estudio de caso. Ciencias de la Información. Nieves Lahaba, y.León Santos, M. (2001). La gestión del conocimiento: una nueva perspectiva en la gerencia de las organizaciones. Acimed, 9 (2), pp.121-126. Nonaka, L. y Takeuchi, H (1995). The knowledge-creating company. New York: Oxford University. Nonaka, L., Takeuchi, H., y Umemoto, K. (1996). A theory of organizacional knowledge creation. International Journal of Technology Management, 11 (7-8), pp. 833-845. Pérez Lindo, A. (2005). Gestión del conocimiento. Un nuevo enfoque aplicable a las organizaciones y a la universidad, conocimiento y reconstrucción nacional. Buenos Aires: Editorial Norma. Pérez Lindo, A (1996). Mutaciones. Escenarios y filosofías del cambio de mundo. Buenos Aires: Biblos Pérez Lindo, A. (2009). ¿Para que educamos hoy? Buenos Aires. Disponible en: http://www. augustoperezlindo.com.ar/docs/educacion/para\%20que\%20educamos\%20hoy.pdf. Recuperado el: 09 de junio 
de 2015. Pérez Lindo, A. (2003). Universidad, conocimiento y reconstrucción nacional. Buenos Aires: Biblos. Probst, G., Raub, S. y Romhardt, K. (2001). Administre el conocimiento. México: Prentice Hall. Rodríguez, A., Araujo De La Mata, A. y Urrutia, J. (2001) La gestión del Conocimiento científico-técnico en la universidad: un caso y un proyecto. Cuaderno de Gestión. Vol.1 №1. Universidad del País Vasco. Santos Rego, M. A. (ed.) (2013). Cosmopolitismo y educación. Aprender y trabajar en un mundo sin fronteras (Valencia, Brief, p. 221) Schön, D. A. (1992). La formación de profesionales reflexivos: hacia un nuevo diseño de la enseñanza y el aprendizaje en las profesiones. Barcelona: Paidós. Serrano, J. M., \& Calvo, M. T. (1996). El aprendizaje cooperativo. Psicología de la instrucción I. Variables y procesos básicos. (pp. 217-244). Slavin, R. E., \& Johnson, R. T. (1999). Aprendizaje cooperativo: teoría, investigación y práctica. Buenos Aires: Aique. Toffler, A. (1990). El cambio de poder. Barcelona: Plaza y Janés.

Abstract:

Design projects are individual, creative, and personal, and generate much emphasis on teaching, learning, and teacher's evaluation of the group that must respect the personal style of each student. The goal is to encourage multiple and different aesthetics, accept diversities and cross-cultural influences, and thus identify in projects cultural imprints to nurture and generate more original designs that achieve a high level of competitive success. From these ideas, a specific technique for the design areas in which projects are the backbone and guideline of the training of students was generated. This technique is called Creative Cooperative Challenge (DCC)

Key words:

project - learning - cooperation - creativity - interculture - design - challenge.

\section{Resumo:}

No design, os projetos são individuais, criativos e pessoais gerando muita ênfase no ensino, a aprendizagem e a correção grupal do professor, que deve respeitar o estilo pessoal de cada estudante. O sentido é fomentar as miradas múltiplas e diferentes, aceitar as diversidades e influencias inter-culturais e assim citar nas produções os rastros culturais para nutrir e gerar desenhos mais originais para aceder a maior competitividade. A partir destas idéias se gerou uma técnica específica para as áreas do design onde os projetos são os eixos centrais e critério da formação dos estudantes. Esta técnica é chamada Desafio Criativo Cooperativo (DCC).

Palavras chave:

projeto - aprendizagem - cooperativo - criatividade - inter-culturalidade - design - desafio.

Interculturalidad: miradas múltiples. Desafío Creativo Cooperativo fue publicado de la página 85 a página97 en Cuadernos del Centro de Estudios de Diseño y Comunicación № 64 